\title{
Speeding up the study of diluted dipolar systems
}

\author{
D. A. Martin, ${ }^{1, *}$ T. S. Grigera,${ }^{2,3}{ }^{\dagger} \dagger$ and V.I. Marconi ${ }^{4} \ddagger$ \\ ${ }^{1}$ Instituto de Investigaciones Físicas de Mar del Plata (IFIMAR), \\ CONICET and Universidad Nacional de Mar del Plata, \\ Funes no. 3350, 7600, Mar del Plata, Argentina \\ ${ }^{2}$ Instituto de Física de Líquidos y Sistemas Biológicos (IFLYSIB), \\ CONICET and Facultad de Ciencias Exactas, Universidad Nacional de La Plata, \\ Calle 59 no. 789, B1900BTE La Plata, Argentina \\ ${ }^{3}$ CCT CONICET La Plata, Consejo Nacional de Investigaciones Científicas y Técnicas, Argentina \\ ${ }^{4}$ FaMAF and IFEG (UNC-CONICET), Universidad Nacional de Córdoba, X5000HUA Córdoba, Argentina
}

(Dated: December 18, 2018)

\begin{abstract}
We study the regimes of a diluted dipolar system through Monte Carlo numerical simulations in the $N V T$ ensemble. In order to accelerate the dynamics, several approximations and speed-up algorithms are proposed and tested. In particular, it turns out that "Cluster Move Monte Carlo" algorithm speed-up to two decades faster than traditional Monte Carlo, depending on temperature and density. We find simple-fluid, chain-fluid, ring-fluid, gel and antiparallel columnar regimes, which are studied and characterized through positional, orientational and thermodynamical observables.
\end{abstract}

PACS numbers: $64.75 . \mathrm{Yz}, 75.50 . \mathrm{Mm}, 64.60 .-\mathrm{i}$

\section{INTRODUCTION}

Over the last decade, the study of self-assembling diluted systems has received renewed attention from the experimental, theoretical and numerical points of view. In particular, particles with anisotropic interactions and interaction energy of the order of $k_{B} T$ (where $k_{B}$ is Boltzmann's constant and $T$ is the temperature) are now being synthesized $[1,2]$ and promissory applications have been proposed for them [3-6]. Examples of those kind of systems include colloidal suspensions of dipolar particles, particles with multipolar behavior [7], patchy particles [8], or Janus particles [9].

The simplest particles with anisotropic interaction are perhaps those endowed with a spherical hard core and a dipole moment. Diluted dipolar systems (DDSs) have been extensively studied through numerical simulations [10-25], which include simple, gas-like order, chain-fluid $[10,11,24]$, ring-fluid $[17,24]$, gel $[12,22]$, and columnar structures with crystal-like order [15, 20, 21]. Related studied systems include discotic liquid crystals [26], dipolar spheres interacting with nonmagnetic rods [27], simplified dipole-like interaction models [15], binary mixtures of anisotropic particles [28] and 2-D systems [19]. Those systems also display rich regime diagrams.

A major issue with simulations of diluted systems is the extraordinary high computational cost, even for small systems (one thousand particles), which has led to the development of several speed-up strategies [29-31]. Here we introduce the Cluster Move Monte Carlo (CMMC) algorithm to speed-up study of diluted self-assembling systems. For a wide range of temperatures and densities,

\footnotetext{
* dmartin@ifimar-conicet.gob.ar

$\dagger$ tgrigera@iflysib.unlp.edu.ar

‡vmarconi@famaf.unc.edu.ar
}

CMMC turns out to be faster than previously proposed algorithms by a factor about 10 in most cases, and up to 100 at some temperatures and densities, see Supplemental Material (SM) at [URL will be inserted by publisher] for details on speed-up factors. The algorithm is tested in a diluted system of simple dipolar particles, exploring various regimes. CMMC do allows us to study much lower temperature and density regimes. Using CMMC, we are able to describe a hard to find antiparallel columnar regime with crystal-like order in the very diluted low temperature region among other well known regimes.

The article is organized as follows: Sec. II presents the Cluster Move Monte Carlo algorithm. Sec. III gives details of the dipolar model under study. Evidence of the speed improvement brought about by CMMC at low densities and temperatures is presented in Sec. IV. In Sec. $\mathrm{V}$ we show the different structural regimes that arise varying temperature and density. Finally we collect all results to present a diagram with the different regimes in Sec. VII, together with the conclusions.

\section{CLUSTER MOVE MONTE CARLO}

The algorithm is designed for the simulation of diluted self assembling particles. In this work, the algorithm is tested on an off-lattice system of spherical particles with diameter $\sigma$. The algorithm employs classical singleparticle Monte Carlo (MC) moves (displacement and rotation) together with movements of groups of particles (clusters).

To define a cluster, two particles are considered neighbors if they are separated by a distance $r_{N}=\sqrt{1.5} \sigma$ or smaller (notice that alternative definitions have been proposed [17]). A cluster is then defined as the smallest set of particles such that if particle $i$ belongs to a cluster, all neighbors of particle $i$ belong to the same cluster. 
Each particle belongs to exactly one cluster; there may be clusters with only one particle.

CMMC trial moves are:

1. With probability $1-p_{\mathrm{CM}}$, a single-particle movement is proposed. This movement attempt is either one translation, with probability $p_{T}$, a rotation with probability $p_{R}$ or a full movement (rotation plus translation), with probability $p_{R T}$ $\left(p_{T}+p_{R}+p_{R T}+p_{\mathrm{CM}}=1\right)$. The relative values of $p_{T}, p_{R}$ and $p_{R T}$ are modified every $250 \mathrm{MC}$ steps, using an algorithm that favors successful trials. In practice, $p_{T}, p_{R}$ and $p_{R T}$ are always larger than 0.1 , they evolve on the first $10^{5} \mathrm{MC}$ steps, and have negligible changes for larger times.

2 . With probability $p_{\mathrm{CM}}$, a cluster move is proposed. A cluster is selected and displaced uniformly to a random location in the simulation box. To ensure detailed balance, the move is rejected if it results in a change in the number of clusters. We have used $p_{\mathrm{CM}}=0.001$. Similar values give similar speed-ups.

Trial moves are then accepted or rejected as usual with the Metropolis criterion, i.e. they are accepted with probability $p=\min [1, \exp (-\beta \Delta U)]$, where $\Delta U$ is the change in the system total energy, as a result of the proposed move, and $\beta=1 / k_{B} T$.

Variants of CMMC, adding cluster rotations and cluster inversion (inversion of all dipole moments of the cluster) were explored. However no further acceleration was found adding those trial moves to the cluster displacement.

We compare the performance of CMMC with standard MC and with the aggregation volume bias (AVB) algorithm specialized for dipolar systems as described by Rovigatti, Russo and Sciortino [17, 18]. This algorithm uses two kinds of trial moves, performed as follows. Within AVB, a particle $i$ is chosen at random and the simulation volume is divided in two fractions, $V_{\text {in }}$, the positions close to $i$ where another particle $j$ is considered bounded (roughly speaking, they are neighbors and their interaction energy is large and negative), and $V_{\text {out }}$ (the rest of the simulation box). Then either an in $\rightarrow$ out (with probability $x$ ) or an out $\rightarrow$ in (with probability $1-x)$ trial move is proposed. For the in $\rightarrow$ out case, a particle $j \neq i$ within $V_{\text {in }}$ is chosen and a random position within $V_{\text {out }}$ and a random orientation are proposed. The out $\rightarrow$ in move is proposed similarly [18, 29].

\section{DIPOLAR PARTICLE MODEL}

In order to test the CMMC algorithm, a system of $N$ particles of size $\sigma$ with a point dipole in the center of the particle is studied. More precisely, the potential energy is $[16,24]$ :

$$
U\left(\left\{\mathbf{r}_{i}, \mathbf{p}_{i}\right\}\right)=\sum_{i>j}^{N} U_{0}\left(\mathbf{r}_{i j}, \mathbf{p}_{i}, \mathbf{p}_{j}\right),
$$

where $\mathbf{r}_{i}, \mathbf{p}_{i}$ are the position and dipole moment of particle $i, \mathbf{r}_{i j}=\mathbf{r}_{i}-\mathbf{r}_{j}$ and the pair potential is

$$
\begin{aligned}
U_{0}\left(\mathbf{r}, \mathbf{p}_{1}, \mathbf{p}_{2}\right) & =U_{d}(r)+U_{p}\left(\mathbf{r}, \mathbf{p}_{1}, \mathbf{p}_{2}\right) \\
U_{d}(r) & =\epsilon\left(\frac{\sigma}{r}\right)^{36} \\
U_{p}\left(\mathbf{r}, \mathbf{p}_{1}, \mathbf{p}_{2}\right) & =\left(\frac{\epsilon \sigma^{3}}{p_{0}^{2}}\right) \frac{\mathbf{p}_{1} \cdot \mathbf{p}_{2} r^{2}-3\left(\mathbf{r} \cdot \mathbf{p}_{1}\right)\left(\mathbf{r} \cdot \mathbf{p}_{2}\right)}{r^{5}},
\end{aligned}
$$

where $r=|\mathbf{r}|, \epsilon$ is the energy scale, and all particles have identical dipole moments, $\left|p_{i}\right|=p_{0}$. Simulations are carried out in a periodic cubic box of side $L$ and the average number density is $\rho_{n}=N / V, V=L^{3}$. The solvent is not considered explicitly here (apart from a possible electric permitivity or magnetic permeability included in $\epsilon)$. We report results using the dimensionless quantities $U^{\prime}=U / \epsilon, r^{\prime}=r / \sigma$ (and similarly for all defined lengths), $\mathbf{p}_{i}^{\prime}=\mathbf{p}_{i} / p_{0}, T^{\prime}=k_{B} T / \epsilon, \rho^{\prime}=\sigma^{3} \rho_{n}$. For the sake of notational simplicity we omit the primes from now on.

Notice that several similar models have been proposed, using the same dipole interaction, $U_{p}$, but different spherical short-range potentials, $U_{d}$, such as dipolar hard spheres or the Stockmayer fluid.

Due to the relatively long range of the dipole interaction potential, dipolar systems have been usually studied using Ewald sums [18, 27]. Computationally less demanding alternatives have also been proposed and studied in detail [32-35]. Following [34, 35], in this work we replace $U_{0}$ in Eq. (1) by a shifted and truncated version $U_{S P}$

$$
U_{\mathrm{SP}}\left(\mathbf{r}, \mathbf{p}_{1}, \mathbf{p}_{2}\right)=U_{0}\left(\mathbf{r}, \mathbf{p}_{1}, \mathbf{p}_{2}\right)\left[1-r^{3} / r_{c}^{3}\right]
$$

for $r<r_{c}$, and zero otherwise. We use a cut-off distance $r_{c}=7 \sigma$. For some temperatures and densities we have tested different values of $r_{c}$, ranging from $r_{c}=4$ to $r_{c}=$ 20, as well as the Ewald sums method. We have not seen any qualitative differences for $r_{c}>5$, but we have taken $r_{c}=7$ as a safe choice [16]. The cell indexation method was used to speed-up the interacting neighbors search [36].

\section{ALGORITHM PERFORMANCE}

We have simulated the dipolar particle system with $\mathrm{MC}, \mathrm{ABV}$ and $\mathrm{CMMC}$, using $U_{S P}$ (the potential in Eq. (5)) instead of $U_{0}$, at temperatures $T=0.03,0.035$, $0.04,0.045,0.05,0.06,0.07,0.08,0.09,0.1,0.11,0.12$, $0.13,0.14,0.15,0.16,0.18,0.2,0.24,0.3,0.36$ and densities $\rho=0.35^{3}, 0.25^{3}, 0.2^{3}, 0.125^{3}, 0.1^{3}, 0.07^{3}, 0.05^{3}$ and $0.03^{3}$. Packing fraction $\phi$, can be related to $\rho$ using $\phi=\pi \rho \sigma^{3} / 6$. Unless stated otherwise, we report results for $N=1000$, and $r_{c}=7$.

The performance of standard MC, AVB and CMMC is compared in Figs. 1, 2, and 3.

In Fig. 1, we show energy evolution as a function of MC steps for a system of $N=1000$ particles, average 


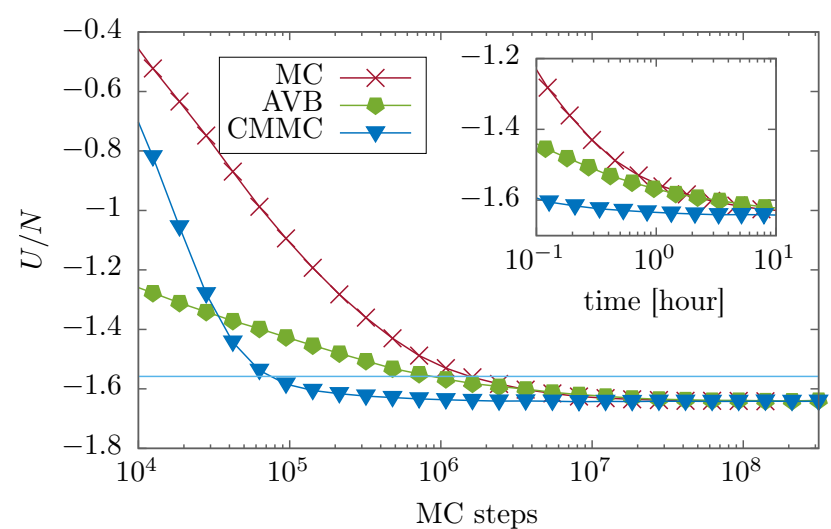

Figure 1. Potential energy per particle as a function of MC steps for $T=0.08, \rho=1 / 8^{3}, N=1000, r_{c}=7 . \quad U$ is computed as in Eq. (1), replacing $U_{0}$ by $U_{S P}$, Eq. (5). A line is drawn at $95 \%$ of asymptotic energy value. Inset: Same results as a function of wall time. Results where found using one core of a 7 th generation Intel i7 computer.

density $\rho=1 / 8^{3}$, temperature $T=0.08$ and cutoff distance $r_{c}=7$. We see that all methods lead to the same asymptotic value. In order to measure the time it takes to reach asymptotic value of energy, we define $\tau_{E}$ as the number of MC steps needed for reaching 95\% of energy asymptotic value. Energy as a function of wall time (the real, physical time) is shown in the inset of that figure. In terms of real time, both AVB and CMMC take less than $10 \%$ more time than MC for performing the same number of MC steps. The only exception is during the first $\simeq 2000 M C$ steps, when there are many solitary particles, and the speed of CMMC is reduced by a factor almost two.

In Fig. 2 we show $\tau_{E}$ for all methods, as a function of $T$. In the inset, we do the same as a function of $\rho$. Both AVB and CMMC considerably speed-up the evolution of the dipolar fluid (compared to standard MC). Adding cluster rotations, dipole inversion or cluster destruction to the cluster displacement move of CMMC does not result in further accelerations. The AVB vs. CMMC comparison shows that AVB performs better at moderate temperatures, while remarkably CMMC is better at low temperatures (CMMC is more than 30 times faster at the lowest temperature we tested, see SM at [URL will be inserted by publisher]).

In Fig. 3, we plot the connected, normalized, energy correlation function $E_{C o r r}(\tau) \doteq$ $\left[\langle E(t) E(t+\tau)\rangle-\langle E(t)\rangle^{2}\right] /\left[\left\langle E(t)^{2}\right\rangle-\langle E(t)\rangle^{2}\right] . \quad E_{C o r r}$ is one for a completely correlated system (for instance, at $\tau=0$ ) and zero for completely uncorrelated ones. We measure it at time $t$ when the system has reached its asymptotic value of energy. In order to measure the time it takes to decorrelate the energy, we define $\tau_{C}$ as the time needed to decorrelate it a $95 \%$ (i.e. $\left.E_{C o r r}\left(\tau_{C}\right)=0.05\right)$. In the inset of Fig. 3 , we plot $\tau_{C}$ as a function of temperature.

Next, we study whether the speed-up algorithm is use-

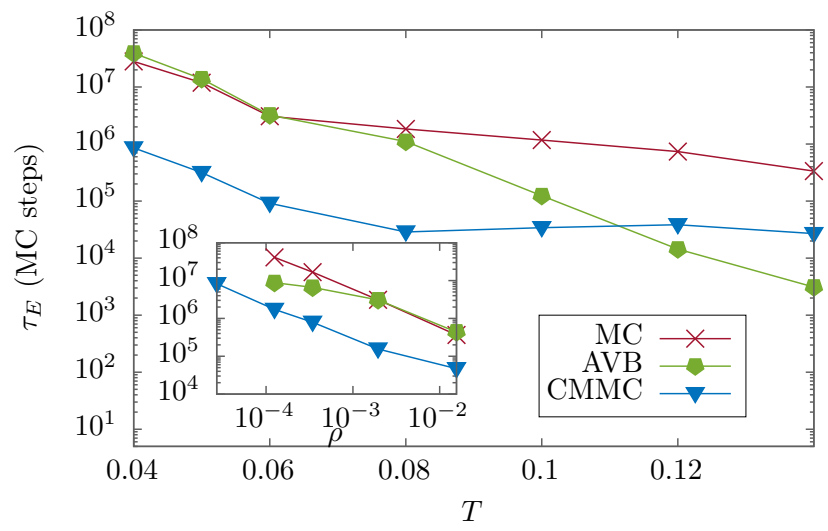

Figure 2. MC steps needed in order to reach $95 \%$ of the asymptotic value of energy, as a function of temperature, $\rho=$ $1 / 8^{3}, N=1000, r_{c}=7$. Inset: Same results as a function of density, for $T=0.08$.

ful for larger system sizes. We have simulated systems of $N=8000, N=27000$ and $N=64000$ particles, at moderate temperature $T=0.08$ and density $\rho=1 / 8^{3}$. We plot the energy as a function of wall time, in Fig. 4. In the inset, we show energy as a function of wall time per particle. We see that CMMC algorithm behaves well on system size (i.e. the wall time needed for performing a MC step is roughly proportional to $N$ ).

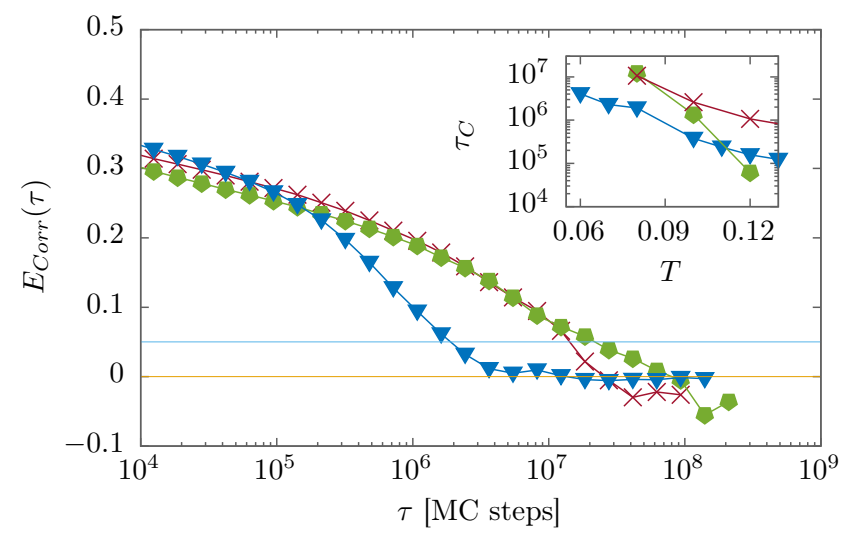

Figure 3. Energy correlation for $T=0.08, \rho=(1 / 8)^{3}, N=$ $1000, r_{c}=7$ using the same symbols as in Fig. 1 . We define the energy correlation time $\tau_{C}$ as the time needed for the normalized correlation function to decay to 0.05 . Inset: $\tau_{C}$ as a function of temperature $\rho=(1 / 8)^{3}$, for different algorithms.

Efficiency of CMMC is due to performing a collective movement in a single step, which would require many single-particle moves to be achieved otherwise. We thus do not expect its efficiency to be sensitive to the truncation distance $r_{c}$. In SM, we show results with and without CMMC, for several values of $r_{c}$, and using Ewald sums. In all cases, CMMC performs at least 10 times faster than MC.

The final states reached with CMMC (the different regimes that will be discussed in more detail in Sec. V) 


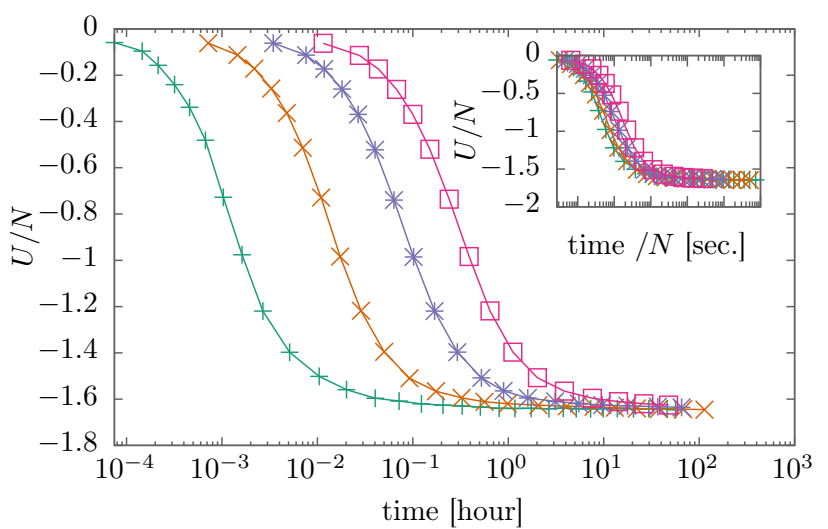

Figure 4. Energy per particle, as a function of wall time, for $T=0.08, \rho=(1 / 8)^{3} r_{c}=7$ and several system sizes. From left to right: $N=1000, N=8000, N=27000$ and $N=64000$. Inset: Same results as a function of wall time per particle. Simulations were performed on a 7 th generation Intel i7 computer.

are very stable within simulation times: all studied observables remain stationary over the last $90 \%$ of the simulation time, and they pass two equilibration tests. First, the derivative of the asymptotic energy with respect to $T$,

$$
C_{v, d}\left(\frac{T_{i}+T_{i+1}}{2}\right)=\frac{U\left(T_{i+1}\right)-U\left(T_{i}\right)}{T_{i+1}-T_{i}}
$$

and the energy fluctuations,

$$
C_{v, f}\left(T_{i}\right)=\frac{\left\langle U^{2}\left(T_{i}\right)\right\rangle-\left\langle U\left(T_{i}\right)\right\rangle^{2}}{T_{i}^{2}}
$$

give compatible results. This is a sign that the simulation has explored a fair portion of the phase space (in equilibrium, $C_{v, d}=C_{v, f}=C_{v}$, where $C_{v}$ is the constantvolume specific heat). In addition, we obtain the same results starting the simulation from either high or low temperature configurations. In typical runs, which we call "cooling" runs, we start from completely random positions and orientations (i.e. a very high temperature configuration) and quench it to temperature $T$ at $t=0$. For "heating" runs, we take a configuration that reaches asymptotic values of all observables at $T=0.04$ and we increase temperature by $\Delta T=0.0025$ every $10^{6} \mathrm{MC}$ steps, while measuring several observables. As shown below in Sec. VB, the values obtained from heating or cooling coincide, with no sign of hysteresis. That is, the algorithm does not face obvious metastability problems. In particular, the values reported below for the crossover temperatures are independent of the procedure (heating or cooling) used to find the different regimes.

\section{REGIMES AND CROSSOVERS}

\section{A. Regimes}

Numerical results allow us to distinguish several different regimes, in terms of the structures the system forms. We describe them first qualitatively. In the next subsection we introduce several observables that are used for defining crossovers between regimes. From high to low temperature, we find:

- Simple-fluid. Corresponds to a homogeneous system of solitary particles (i.e. particles that do not form clusters), with negligible orientation correlations. A snapshot of this regime is shown in Fig. 5(a).

- Complex-fluid. There may be a chain-fluid or a ring-fluid. Those regimes are characterized by the presence of many small clusters and almost uniform density. The system does not percolate. Most particles have exactly two neighbors, they are aligned head-to-tail, and a low but nonzero polarization can be measured (at least in small systems). A ring is a cluster where every particle has exactly two neighbors. A chain (also known as string $[15,16]$ ) is a cluster where every particle has two neighbors, except for two of them (the chain tips), which have exactly one neighbor. We find that chains are more likely at high density, while rings dominate at very low density. Snapshots of the ring-fluid and chainfluid regimes are shown in Figs. 5(b) and 5(c).

- Gel. It is a percolating system. Most particles belong to the same cluster, which may, or may not span the whole simulation box. Most particles have two neighbors, while some of them have more than two. A snapshot of the gel regime is shown in Fig. 6(a).

- Columnar. The antiparallel columnar regime is defined by the presence of a single cluster containing all particles. Each particle has several neighbors, and dipoles are oriented head-to-tail forming a chain which is surrounded by similar chains with opposite orientation (see Fig. 6(b)). Columnar structures show long range crystal-like order (see radial distribution function, Fig. 7). The cluster tends to be elongated in the direction of those chains. It is compact in the sense that all particles are found within a small, high density region, while the rest of the simulation box is empty. We have found this columnar regime at low and high densities. Crystals have been found in previous work $[15,19]$, but in situations where the density was uniform over the simulation box. In our case, the average density is roughly 250 times smaller than the density of the crystal. The columnar shape we find is probably related to the existence of a large 
void region in the simulation box. Our result is similar to the columnar regime of the Stockmayer model [21], which has the same dipolar term, $U_{p}$, but a Lenard-Jones potential for the isotropic term $U_{d}$, i.e. in the Stockmayer model there is an attractive isotropic term in addition to the anisotropic dipolar contribution. Columnar regimes have been found under external applied field, either experimentally, [37, 38], or in 2-D simulations [14].

We find that, at low temperatures and densities (i.e. in the gel or crystal regime), there are high density regions and voids. In order to identify different structures, we define

$$
u(r)=\sum_{i, j} \int_{r}^{r+\Delta r} d V^{\prime} \delta\left(r^{\prime}-\left|\mathbf{r}_{i j}\right|\right) /\left[2(N-1) 4 \pi r^{2} \Delta r\right] .
$$

When the density is homogeneous, $u(r)$ is equal to $\rho g(r)$ $(g(r)$ is the radial distribution function). Examples of $u(r) / \rho$, for several regimes, are shown in Fig. 7. For the simple-fluid, which has homogeneous density, we get a rather simple diagram, which goes to 1 at $r \sim 3$. For all other samples, we have $u(r) \gg \rho$. This is a direct consequence of having high density regions and voids. For the columnar regime, there are several peaks, that extend to typical column widths. In the figure, arrows are drawn at the two-particle minimum energy distance (i.e. the value of $r$ that minimizes Eq. (5)), and integer multiples of that distance.

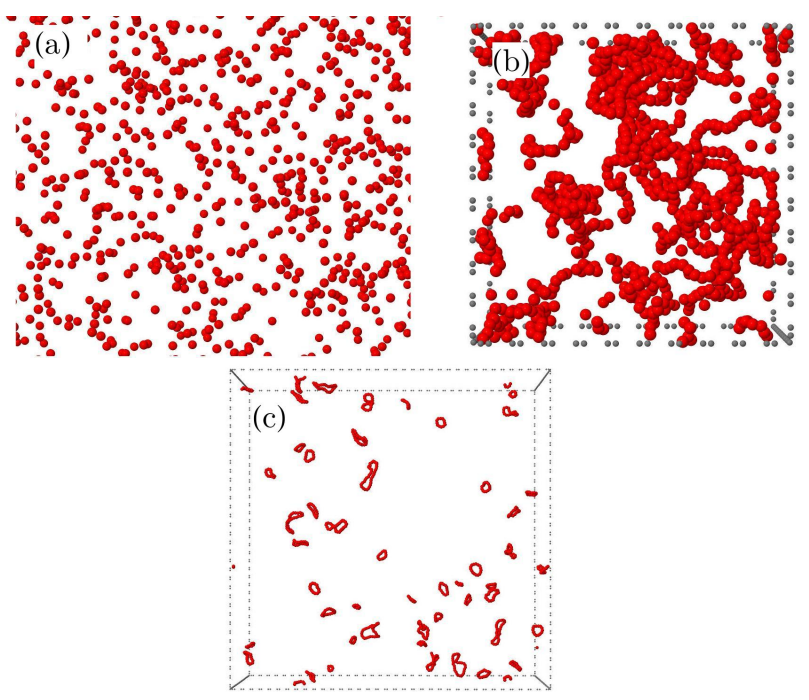

Figure 5. Snapshots of (a) portion of a simple-fluid $\left(\rho=1 / 8^{3}\right.$, $T=0.16$ ), and (b)-(c), complex fluid. In (b), there is a chainfluid sample $\left(\rho=1 / 4^{3}, T=0.14\right)$, while in (c) there is a ring-fluid $\left(\rho=0.07^{3}, T=0.08\right)$ sample.

\section{B. Crossovers}

Here we report observables that can pinpoint the
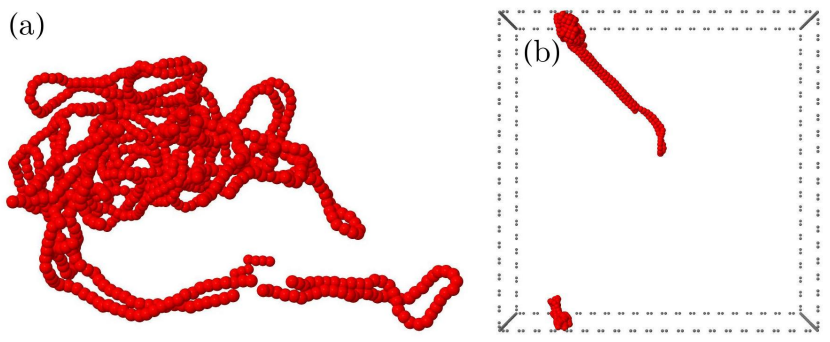

Figure 6. Snapshots of (a) gel including all particles in the simulation $\left(\rho=1 / 8^{3}, T=0.06\right)$, and (b) columnar regime at low density $\left(\rho=1 / 8^{3}, T=0.04\right)$. Snapshots were performed with Jmol [39].

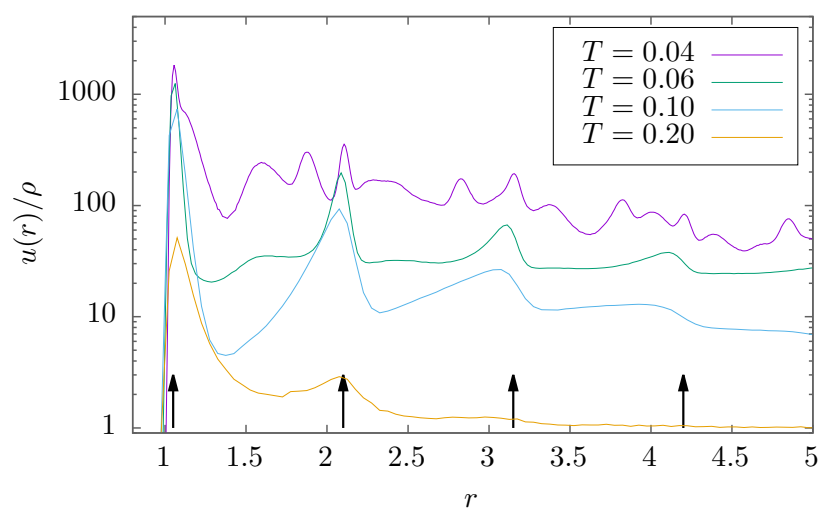

Figure 7. Example of $u(r) / \rho$ at density $\rho=(1 / 8)^{3}$, for different regimes. From top to bottom: columnar $(T=0.04)$, gel $(T=0.07)$, chain-fluid $(T=0.10)$ and simple-fluid $(T=0.20)$.

crossovers between regimes. All results shown are obtained from at least eight independent systems at each density and temperature, and data is collected after energy and energy fluctuations reach a stationary state. The specific heat per particle, $C_{v} / N$, is estimated from energy fluctuations, Eq. (7) and from numerical derivatives of the energy values, Eq. (6). Both methods yield similar results in the studied temperature range, suggesting that the systems have reached equilibrium. Given the well-known equilibration problems in these kind of systems, we do not claim that the regimes and crossovers we describe below correspond to true equilibrium thermodynamic phases. However we do emphasize that they are extremely stable within the simulation times reached, and that we can reach them both from stabilized systems at high or low temperatures as mentioned in sec. IV. We would like to emphasize that we are describing the regimes that in practice show up, in the sense that in simulations of finite but extremely long times they remain stable. Based on our stability tests, we expect those structures to be stable enough to be accessible in poten- 

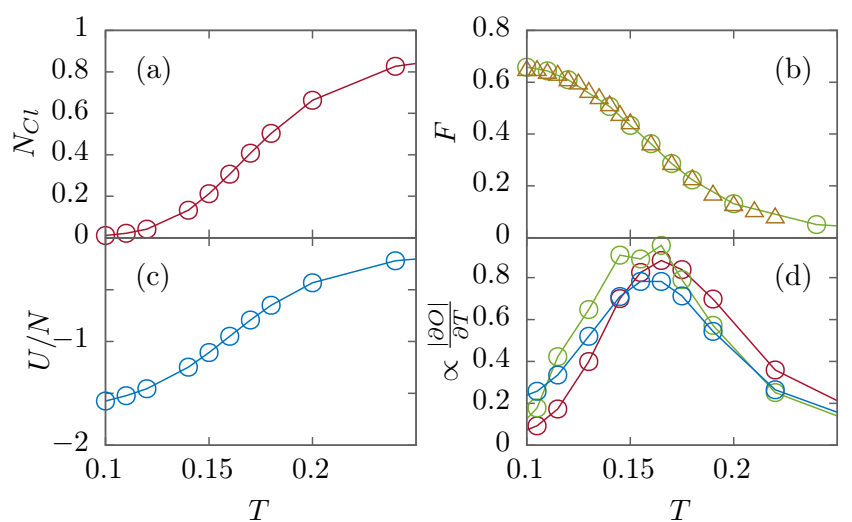

Figure 8. Crossover between simple-fluid and complex-fluid, described using several variables as a function of temperature, for $\rho=1 / 4^{3}$. (a) Number of clusters, (b) local parallel order parameter, Eq. (9)), (c) energy, and (d) normalized derivatives of those variables. In (b), triangles are obtained from heating, while circles are results from cooling.

tial experiments.

We now explain how the crossovers between the different regimes are calculated. In what follows $N_{C l}$ is the number of clusters, $l_{j}$ is the amount of particles in cluster $j$ (cluster size), and $l_{\max }$ the size of the largest cluster.

a. Simple-fluid to Complex-fluid. At a very high temperature, only solitary particles at uniform density are found. This is a simple-fluid. Cooling the system, at some point one starts to detect cluster formation, accompanied by the onset of local parallel order. The number of clusters, $N_{C l}$ displays a sharp decrease on cooling at a temperature roughly coincident with a peak in the specific heat. One measure of the local parallel order is

$$
F=\frac{1}{N} \sum_{i, j} \mathbf{p}_{i} \cdot \mathbf{p}_{j} f\left(r_{i j}\right), \quad f(x)=\frac{1}{x^{2}} H(4-x),
$$

where $H$ is the Heaviside step function. This is similar to the local order parameter described in [20], except that a Heaviside function if used here, in order to reduce the analysis volume. The funcion $f(x)$ is used so that nearest neighbors have more relevance on $F$. Alternative choices of $f(x)$ yield similar results.

Fig. 8 shows the behavior of the number of clusters, the local parallel order parameter, energy, and their derivatives to temperature. The derivatives of all those observables display peaks at similar temperatures. Results for $F$ as a function of temperature are shown for both cooling and heating in Fig. 8(b). They coincide within statistical error, and the same holds for all other observables in the simple-fluid to complex-fluid crossover.

b. Chain-fluid to Ring-fluid. In order to distinguish between chain-fluid and ring-fluid, we measure the number of particles belonging to chains and rings. We consider the system to be in the chain-fluid regime when there are more particles forming chains than particles forming rings, and in the ring-fluid regime if the oppo-

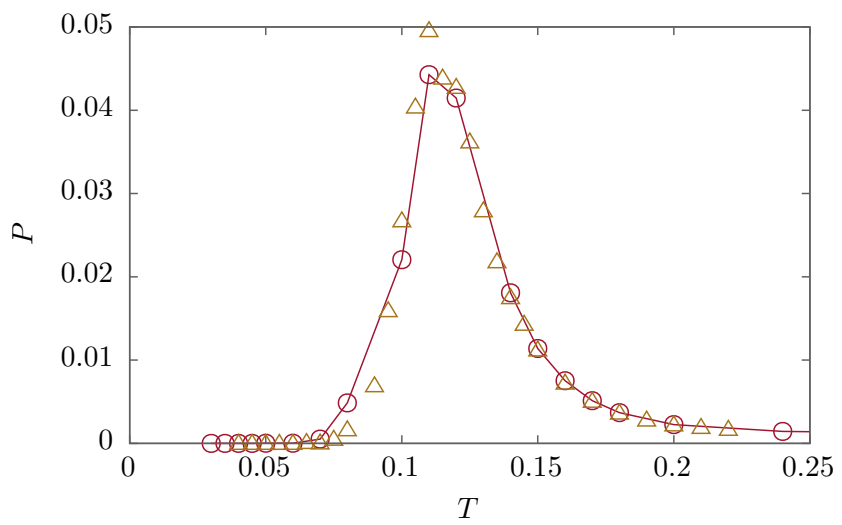

Figure 9. Behavior of the percolation parameter $P$, Eq. (10), versus temperature for $\rho=1 / 4^{3}$. Triangles: heating, circles: cooling.

site is true.

c. Complex-fluid to Gel. Cooling the complex-fluid further, the system forms a single cluster. The most useful observable to detect the fluid-gel crossover is the percolation parameter, also known as (normalized) mean cluster size,

$$
P=\frac{1}{N^{2}} \sum_{j=1, N_{c}-1} l_{j}^{2}
$$

where the sum is over all clusters but the largest. It has a peak at the percolation transition [40, 41]. For a system divided into $n$ equal clusters, $P=(n-1) / n^{2}$ (for a gel, $P=0$, for a simple-fluid $P \simeq 1 / N, P(n=$ $2)=1 / 4, P(n=3)=2 / 9$, and so on $)$. Fig. 9 shows percolation parameter $P$, for both cooling and heating. Both curves show similar behavior, and have a peak at the same temperature. Another related observable that separates complex-fluid from gel is the derivative of $l_{\max }$ with $T$, which presents a peak at the same temperature as $P$.

d. Gel to Columnar. At the lowest studied temperatures we find a previously uncharacterized (to the best of our knowledge) regime where a compact, crystal-like structure, with columnar shape appears. This structure can be distinguished from the gel by computing the number of particles with more than two neighbors $N_{m}$, local antiparallel order $A$, or the local density $N_{n}$ (the density in a sphere of radius 4 , about the particle). Local antiparallel order is measured through

$$
A=\frac{1}{N} \sum_{i, j}\left|\mathbf{p}_{i} \cdot \mathbf{p}_{j} f\left(r_{i j}\right)\right|-\mathbf{p}_{i} \cdot \mathbf{p}_{j} f\left(r_{i j}\right) .
$$

For uncorrelated orientation and position, $A=A_{\infty}=$ $(N-1) \rho / N$, if all dipoles point in the same direction $A=0$, and $A \gg A_{\infty}$ in the antiparallel regime. In Fig. 10 we show values of those observables for $\rho=1 / 4^{3}$. All of them present an abrupt drop around $T=0.05$. The derivatives of those quantities show a (negative) peak at 

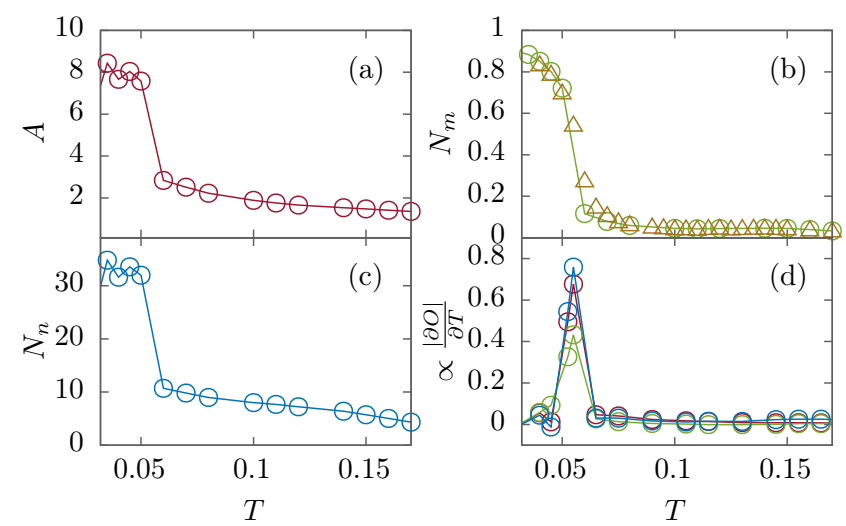

Figure 10. Behavior of some observables as a function of temperature, about the columnar to gel crossover. (a) Local anti-parallel order $A$, (b) number of particles with multiple neighbors (more than two), $N_{m}$, (c) local density $N_{n}$, and (d) the normalized (negative of) the temperature derivative of those observables. In (b), triangles: heating, circles: cooling.

the same temperature (see Fig. 10). We have repeated this method in the whole density range (from $\rho=27 \times$ $10^{-6}$ to $\left.\rho=0.064\right)$. In all cases the peaks take place at the same temperature. Results from heating and cooling coincide. Values of $N_{m}$ obtained from heating and from cooling are both shown in Fig. 10(b).

\section{DISCUSSION AND CONCLUSIONS}

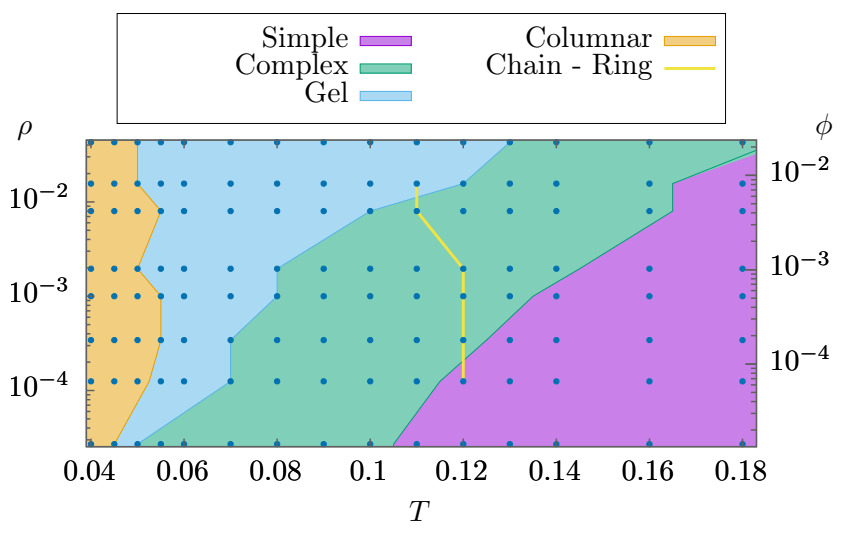

Figure 11. Regime diagram: density versus temperature. From left (low temperature) to right, there are columnar, gel, complex-fluid, and simple-fluid regimes. Boundaries among regions are calculated from the peaks of Figs. 8(d) (simplefluid to complex-fluid) 9 (complex-fluid to gel) and 10(d) (gel to columnar). The complex-fluid is divided into two regions: at high density, chain-fluid is found, while at low density, there is a ring-fluid. A line dividing chain-fluid from ring-fluid is shown. In the right y-label, we have included the packing fraction, $\phi=\pi \rho \sigma^{3} / 6$.

We outline a regime diagram, where we report the tem- perature of the crossovers among the different regimes, detected as discussed in Sec. VB for several densities (from $\rho \sim 3 \times 10^{-5}$ to $\sim 4 \times 10^{-2}$ ) and temperatures (see Fig. 11). We emphasize that boundaries in Fig. 11 have been calculated using the peak values of the observables under consideration. As may be expected from Figs. 8, 9 and 10, there may be a broad coexistence region about the peaks. The study of coexistence regions is beyond the scope of this article.

From Fig. 11, we see that the antiparallel columnar structure is found at low temperatures. The crossover temperature from columnar to gel does not have a strong dependence on $\rho$. At higher temperatures, a gel shows up. The density region where a gel is available decreases on diluting. It would be interesting to study whether gel regime disappears at low enough density. The gel-fluid crossover temperature grows with $\rho$. At higher temperatures, a complex-fluid is found. It may be a chain or a ring fluid. Chains are more likely to take place at high density, whereas rings dominate at very low density. Finally, there is a simple-fluid at high temperature. The complex-fluid to simple-fluid crossover temperature also grows with $\rho$.

Results in Fig. 11 are for $r_{c}=7$. We expect to find the same regimes for other values of $r_{c}$. We have run simulations for several values of $r_{c}$ and with Ewald sums. We have found compatible structural results. In all cases, CMMC speeds-up 1 to 2 orders of magnitude. Our choice of short-range potential, $U_{d} \propto r^{-36}$ represents hard spheres in such way that $U_{d}$ and all its derivatives are continuous, and may be reproduced under other dynamics such as molecular dynamics. We have also tested dipolar soft spheres (DSS, $U_{d} \propto r^{-12}$ ), and dipolar hard spheres (DHS). CMMC considerably speeds-up dynamics for DHS and DSS. See SM at [URL will be inserted by publisher] for a description of results using other values of $r_{C}$, Ewald sums, DSS and DHS.

In defining the above-mentioned regimes we have combined positional, orientational, and thermodynamic observables. In most cases, there is more than one observable capable of detecting the given crossover, at the same temperature. For instance, the temperature and density range where large fluctuations in local density take place is similar to the temperature-density range where antiparallel orientational order takes place.

Numerical studies of dipolar particles use similar potentials to the ones used here. Temperature and density can be compared in terms of the ratio $T^{\prime}$ between $k_{B} T$ and typical energy value of two nearest neighbor particles, $\epsilon$. For instance, other studies finding columnar structures in simplified dipolar-like particles [15, 19], would have effective temperatures and densities in the range $0.1<T^{\prime}<2$ and $0.1<\rho^{\prime}<1.4$. Studies of ring formation $[18,24]$ were performed on densities about $\rho \geq 0.005$, and temperatures $T^{\prime}>0.125$. The analysis performed here considers temperatures as low as $T^{\prime}=0.04$ and densities as low as $2.7 \times 10^{-5}$, considerably extending the regime diagram. 
Magnetic nanoparticles consisting on iron oxides can be easily synthesized. They have typical sizes of $\sigma \sim$ $10 \mathrm{~nm}$, and consist of a single domain crystal. Iron oxides have typical magnetization per volume, of $M_{0} \sim$ $3-410^{5} \mathrm{~A} / \mathrm{m}$. Thus, the largest dipole-dipole energy between two particles is $U=\mu / 4 \pi M_{0}^{2}(\pi / 6)^{2} \sigma^{3}$, which gives $3.3510^{-21} J$ in water. This gives $k_{B} T / \epsilon \sim 1.3$ at room temperature. If particles are coated with a nonmagnetic material of width $d$, former formula reduces to $U=\mu / 4 \pi M_{0}^{2}(\pi / 6)^{2} \sigma^{6}(\sigma+2 d)^{3}$. Recently, $\sigma \simeq 20 \mathrm{~nm}$ particles covered by a $2 \mathrm{~nm}$ surfactant layer have been synthesized [1], with a reported value of $T^{\prime}=k_{B} T / \epsilon \simeq$ $1 / 9$. This results are compatible with single domain nanoparticles. Single domain, cubic magnetite particles with $\sigma \simeq 76 \mathrm{~nm}[2]$ have also been synthesized. Moreover, the theoretical limit for single domain iron oxide nanoparticle size is about $\sigma=128 \mathrm{~nm}$ for spherical magnetite particles [42], which would give, without coating, values of $T^{\prime}=k_{B} T / \epsilon \simeq 0.0008$. Conversely, those particles could be coated, forming, for instance, a $500 \mathrm{~nm}$ size particle, with $T^{\prime}=0.05$. This means that regimes such as the ones described here, should be found in nowadays experiments. A more quantitative relation among theoretical and experimental temperatures and densities, should consider other factors, such as surface tension, or non-equilibrated surface charges, which exceeds the scope of this article.

In conclusion, we have presented an algorithm
(CMMC) that speeds-up simulations of Dipolar Diluted Systems by a factor of 10 to 100, compared to other algorithms, at low temperatures and densities. The algorithm is easy to implement and it does reduce both the equilibration and relaxation time. The physical time it consumes scales almost linearly with system size. Using it, we were able explore lower densities and temperatures, where we find and characterize columnar structures in the extremely diluted dipolar system. The CMMC algorithm can be combined with several speed-up algorithms, such as AVB, Umbrella Sampling and Parallel Tempering. Thus, it appears as a useful and potentially powerful tool for the study of DDs, but also other diluted self-assembling [7-9] or aggregating systems [43].

Finally, several numerical [44, 45] and experimental studies include permanent or time-dependent fields. It would be very interesting to analize the efficiency of this algorithm under such circumstances.

\section{ACKNOWLEDGEMENTS}

We would like to thank Ernesto Loscar for inspiring contributions on percolation. We acknowledge financial support from ANPCyT projects PICT-Joven 3874 and PICT-0735, CONICET and SECyT-UNC.
[1] Mark Klokkenburg, Ben H. Erné, Johannes D. Meeldijk, Albrecht Wiedenmann, Andrei V. Petukhov, Roel P. A. Dullens, and Albert P. Philipse, "In situ imaging of field-induced hexagonal columns in magnetite ferrofluids," Phys. Rev. Lett. 97, 185702 (2006).

[2] Qing Li, Christina W. Kartikowati, Shinji Horie, Takashi Ogi, Toru Iwaki, and Kikuo Okuyama, "Correlation between particle size/domain structure and magnetic properties of highly crystalline fe3o4 nanoparticles," Sci. Rep. 7, 9894 (2017)

[3] Wei-xian Zhang, "Nanoscale iron particles for environmental remediation: An overview," J. Nanopart. Res. 5, 323-332 (2003).

[4] R.A. Crane and T.B. Scott, "Nanoscale zero-valent iron: Future prospects for an emerging water treatment technology," J. Hazzard. Mat. 211-212, 112 - 125 (2012).

[5] Gunjan Verma and P. A. Hassan, "Self assembled materials: design strategies and drug delivery perspectives," Phys. Chem. Chem. Phys. 15, 17016-17028 (2013).

[6] Fernando Martinez-Pedrero, Andrejs Cebers, and Pietro Tierno, "Dipolar rings of microscopic ellipsoids: Magnetic manipulation and cell entrapment," Phys. Rev. Applied 6, 034002 (2016).

[7] Masatoshi Toda, Shinji Kajimoto, Shuichi Toyouchi, Toshihiro Kawakatsu, Yohji Akama, Motoko Kotani, and Hiroshi Fukumura, "Phase behavior of a binary fluid mixture of quadrupolar molecules," Phys. Rev. E 94, 052601 (2016).
[8] Amar B. Pawar and Ilona Kretzschmar, "Fabrication, assembly, and application of patchy particles," Macromol. Rapid. Commun. 31, 150-168 (2010).

[9] Andreas Walther and Axel H. E. Müller, "Janus particles: Synthesis, self-assembly, physical properties, and applications," Chem. Rev. 113, 5194-5261 (2013).

[10] Dongqing Wei and G. N. Patey, "Orientational order in simple dipolar liquids: Computer simulation of a ferroelectric nematic phase," Phys. Rev. Lett. 68, 2043-2045 (1992).

[11] J. J. Weis and D. Levesque, "Chain formation in low density dipolar hard spheres: A Monte Carlo study," Phys. Rev. Lett. 71, 2729-2732 (1993).

[12] T. Tlusty and S. A. Safran, "Defect-induced phase separation in dipolar fluids," Science 290, 1328-1331 (2000).

[13] J. M. Tavares, J. J. Weis, and M. M. Telo da Gama, "Quasi-two-dimensional dipolar fluid at low densities: Monte Carlo simulations and theory," Phys. Rev. E 65, 061201 (2002)

[14] J.-J. Weis, "Low density quasi-two-dimensional dipolar hard spheres in an external field," Mol. Phys. 103, 7-10 (2005).

[15] Amit Goyal, Carol K. Hall, and Orlin D. Velev, "Phase diagram for stimulus-responsive materials containing dipolar colloidal particles," Phys. Rev. E 77, 031401 (2008).

[16] Verónica I. Marconi, Pablo Serra, and Marcelo A. Carignano, "Kinetics of string-gel formation in a system of dipolar colloidal particles," 369, 351-358 (2010). 
[17] Lorenzo Rovigatti, John Russo, and Francesco Sciortino, "No evidence of gas-liquid coexistence in dipolar hard spheres," Phys. Rev. Lett. 107, 237801 (2011).

[18] Lorenzo Rovigatti, John Russo, and Francesco Sciortino, "Structural properties of the dipolar hard-sphere fluid at low temperatures and densities," Soft Matter 8, 63106319 (2012).

[19] Heiko Schmidle, Carol K. Hall, Orlin D. Velev, and Sabine H. L. Klapp, "Phase diagram of two-dimensional systems of dipole-like colloids," Soft Matter 8, 1521-1531 (2012).

[20] V. N. Blinov and V. L. Golo, "Local orientational order in the stockmayer model," JETP Letters 96, 475-479 (2012).

[21] V. N. Blinov, "Columnar phase in the stockmayer model," JETP 117, 343-348 (2013).

[22] Aparna Sreekumari and Patrick Ilg, "Slow relaxation in structure-forming ferrofluids," Phys. Rev. E 88, 042315 (2013).

[23] Lorenzo Rovigatti, José Maria Tavares, and Francesco Sciortino, "Self-assembly in chains, rings, and branches: A single component system with two critical points," Phys. Rev. Lett. 111, 168302 (2013).

[24] Mariano Exequiel Brito, "Dipolar colloid system: phases, gelification dynamics ahd structural characterization," Master Thesis, FaMAF, Universidad Nacional de Córdoba, Argentina, 2014 1, 134501 (2014); M. E. Brito, M. A. Carignano, and V. I. Marconi, Unpublished (2018).

[25] Michela Ronti, Lorenzo Rovigatti, Jose M. Tavares, Alexey O. Ivanov, Sofia S. Kantorovich, and Francesco Sciortino, "Free energy calculations for rings and chains formed by dipolar hard spheres," Soft Matter 13, 78707878 (2017).

[26] Tushar Kanti Bose and Jayashree Saha, "Monte carlo simulations of spontaneous ferroelectric order in discotic liquid crystals," Phys. Rev. Lett. 110, 265701 (2013).

[27] Stavros D. Peroukidis and Sabine H. L. Klapp, "Spontaneous ordering of magnetic particles in liquid crystals: From chains to biaxial lamellae," Phys. Rev. E 92, 010501 (2015).

[28] Amit Goyal, Carol K. Hall, and Orlin D. Velev, "Selfassembly in binary mixtures of dipolar colloids: Molecular dynamics simulations," J. Chem. Phys. 133, 064511 (2010).

[29] Bin Chen and J. Ilja Siepmann, "A novel Monte Carlo algorithm for simulating strongly associating fluids: Applications to water, hydrogen fluoride, and acetic acid," J. Phys. Chem. B 104, 8725-8734 (2000).
[30] Bin Chen and J. Ilja Siepmann, "Improving the efficiency of the Aggregation-vVlume-Bias Monte Carlo algorithm," J. Phys. Chem. B 105, 11275-11282 (2001).

[31] Scott Wierzchowski and David A. Kofke, "A generalpurpose biasing scheme for Monte Carlo simulation of associating fluids," J. Chem. Phys. 114, 8752-8762 (2001).

[32] Christopher J. Fennell and J. Daniel Gezelter, "Is the Ewald summation still necessary? pairwise alternatives to the accepted standard for long-range electrostatics," J. Chem. Phys. 124, 234104 (2006).

[33] Billy W. McCann and Orlando Acevedo, "Pairwise alternatives to Ewald summation for calculating long-range electrostatics in ionic liquids," J. Chem. Theory Comput. 9, 944-950 (2013).

[34] Madan Lamichhane, J. Daniel Gezelter, and Kathie E. Newman, "Real space electrostatics for multipoles. I. Development of methods," J. Chem. Phys. 141, 134109 (2014).

[35] Madan Lamichhane, Kathie E. Newman, and J. Daniel Gezelter, "Real space electrostatics for multipoles. II. Comparisons with the ewald sum," J. Chem. Phys. 141, 134110 (2014).

[36] Mike P Allen and Dominic J Tildesley, Computer Simulation of Liquids (Oxford University Press, 1989).

[37] Y. Lalatonne, J. Richardi, and M. P. Pileni, "Van der Waals versus dipolar forces controlling mesoscopic organizations of magnetic nanocrystals," Nat. Mater. 3, 121 -125 (2004).

[38] N. S. Susan Mousavi, Sachin D. Khapli, and Sunil Kumar, "Direct observations of field-induced assemblies in magnetite ferrofluids," J. Appl. Phys. 117, 103907 (2015).

[39] Angel Herráez, "Biomolecules in the computer: Jmol to the rescue," Biochem. Mol. Biol. Educ. 34, 255-261.

[40] E. S. Loscar and E. V. Albano, "Numerical study of the evaporation/condensation phase transition of droplets for an irreversible reaction model," Europhys. Lett. 85, 30004 (2009).

[41] Dietrich Stauffer and Ammon Aharony, Introduction To Percolation Theory: Revised Second Edition (Taylor \& Francis, 2014).

[42] Diandra L. Leslie-Pelecky and Reuben D. Rieke, "Magnetic properties of nanostructured materials," Chem. Mater. 8, 1770-1783 (1996).

[43] D. A. Weitz and M. Oliveria, "Fractal structures formed by kinetic aggregation of aqueous gold colloids," Phys. Rev. Lett. 52, 1433-1436 (1984).

[44] E.L. Pollock and B.J. Alder, "Static dielectric properties of stockmayer fluids," Phys. A 102, 1 - 21 (1980).

[45] Patrick Ilg and Emanuela Del Gado, "Non-linear response of dipolar colloidal gels to external fields," Soft Matter 7, 163-171 (2011). 